\title{
Studying the Characteristics of a Rectangular Waveguide using HFSS
}

\author{
H. Srikanth Kamath \\ Assistant Professor \\ Department of Electronics and \\ Communication \\ Manipal Institute of Technology, \\ Manipal
}

\author{
Rachit Arora \\ Student \\ Department of Electronics and \\ Communication \\ Manipal Institute of Technology, \\ Manipal
}

\author{
Vriti Agarwal \\ Student \\ Department of Electronics and \\ Communication \\ Manipal Institute of Technology, \\ Manipal
}

\begin{abstract}
The Rectangular waveguides are the earliest mode of transmission lines used for compact systems like radars and inside equipment shelter. An air filled Rectangular waveguide WR-90 is simulated using HFSS simulation software to obtain different parameters. The electric and magnetic field patterns have been analyzed, also intrinsic impedance and wavelength for the first four modes of the waveguide are obtained. First three modes $\left(\mathrm{TE}_{01}, \mathrm{TE}_{10}, \mathrm{TE}_{20}\right)$ are included in the analysis. Characteristic profiles are analyzed and the performance of the waveguide is studied. Theoretical analyses have been studied and both the results have been compared and verified. In addition to that the cutoff wavelength has been determined and compared with the theoretical value and its importance has been described which can be helpful in fabricating the real world components.
\end{abstract}

\section{Keywords}

Rectangular Waveguide, HFSS, TE modes, Field Patterns.

\section{INTRODUCTION}

Rectangular Waveguides are used to transfer electromagnetic energy or power from one point in the space to another efficiently. They are used in many applications such as radars, isolators, attenuators and slotted lines.

An open ended rectangular waveguide WR-90 is taken in an enclosed conducting form. [1], [2] .The medium for transmission is taken as air and the walls should be conducting. It is possible to propagate various modes of electromagnetic waves in a rectangular waveguide. Usually there are three modes of transmission namely TE, TM, TEM but the rectangular waveguide supports TE, TM and not TEM because a unique voltage cannot be defined since there is only one conductor in a rectangular waveguide. A rectangular waveguide cannot propagate below a certain frequency which is called the cutoff frequency. The dominant mode in the rectangular waveguide is $\mathrm{TE}_{10}$ which has the lowest cutoff frequency. [3]- [5].

The simulation software that has been used to simulate the rectangular waveguide parameters is Ansoft HFSS 13.0.This tool is used to simulate complex 3D geometries using Finite element method to compute electrical behavior of high speed and high frequency components. The HFSS most accurately characterizes the electrical performance of the components and effectively evaluates various parameters. It helps the user to analyze the various properties of electromagnetic structures such as S parameters, propagation constant, input impedance, radiation pattern etc. It is a powerful post-processor for unprecedented insight into the electrical performance and the results are more accurate and helpful before the designing of any of the components.
In this paper the characteristics of the rectangular waveguide are determined by simulating through HFSS. The cutoff frequency, radiation pattern and other near field patterns are analyzed using this software [6]-[9].

\section{MATHEMATICAL COMPUTATIONS}

A hollow rectangular waveguide is considered which is situated in the rectangular coordinate system in which its breadth is along the $\mathrm{x}$-axis and length along the $\mathrm{y}$-axis, with inner dimensions as $\mathrm{a} * \mathrm{~b}(\mathrm{a}>\mathrm{b})$. Air is used as the dielectric medium. The electromagnetic waves travel in the $\mathrm{z}$ direction which implies that the $\mathrm{H}$ component of the magnetic field $\mathrm{H}_{\mathrm{z}}$ must exist for the energy transmission in the waveguide. The electromagnetic waves inside the waveguide have various field patterns which can propagate independently and are called modes. It is convenient to classify two-dimensional fields as transverse magnetic (TM) or transverse electric (TE) according to whether $\mathrm{E}$ or $\mathrm{H}$ was transverse to the direction of propagation (or decay). The $\mathrm{TE}_{\mathrm{mn}}$ mode is characterized by $\mathrm{E}_{z}$

$=0$, i.e. the $\mathrm{H}_{\mathrm{z}}$ component must exist for the transmission in the waveguide. In $\mathrm{TM}_{\mathrm{mn}}$ modes $\mathrm{H}_{\mathrm{z}}=0$, and also the $\mathrm{E}_{\mathrm{z}}$ component must exist for the transmission in the waveguide.

The wave equations for wave propagating along the $\mathrm{z}$-axis are given by:

$$
\begin{aligned}
& r=\sqrt{((R+j \omega L)(R+j \omega C))} \\
& r=\alpha+j \beta \\
& \nabla^{2} H_{2}=\mu \varepsilon \frac{\partial^{2} H z}{\partial t^{2}} \text { and } E_{2}=0 \text { for TE waves (I) } \\
& \nabla^{2} E_{2}=\mu \varepsilon \frac{\partial^{2} E z}{\partial t^{2}} \text { and } H_{2}=0 \text { for TM waves (2) }
\end{aligned}
$$

Here $\mathrm{H}_{\mathrm{z}}$ and $\mathrm{E}_{\mathrm{z}}$ are the respective magnetic and electric $\mathrm{z}$ components of the electromagnetic wave inside the waveguide. For the simulation of the rectangular waveguide the dimensions $\mathrm{a}=2.286 \mathrm{~cm}, \mathrm{~b}=1.016 \mathrm{~cm}$ and the propagation constant as 1 is considered. For a TM M,N mode the cutoff frequency, which is defined as the minimum frequency required for the transmission of waves inside the waveguide, is same as the TE mode. The only difference is that one cannot have $\mathrm{m}=0$ or $\mathrm{n}=0$. The mode having the lowest frequency in a particular mode is called the dominant mode. The dominant mode in the rectangular waveguide is $\mathrm{TE}_{10}$ mode which is usually used for all the transmissions in the rectangular waveguide. 


\section{DESIGNING EQUATION}

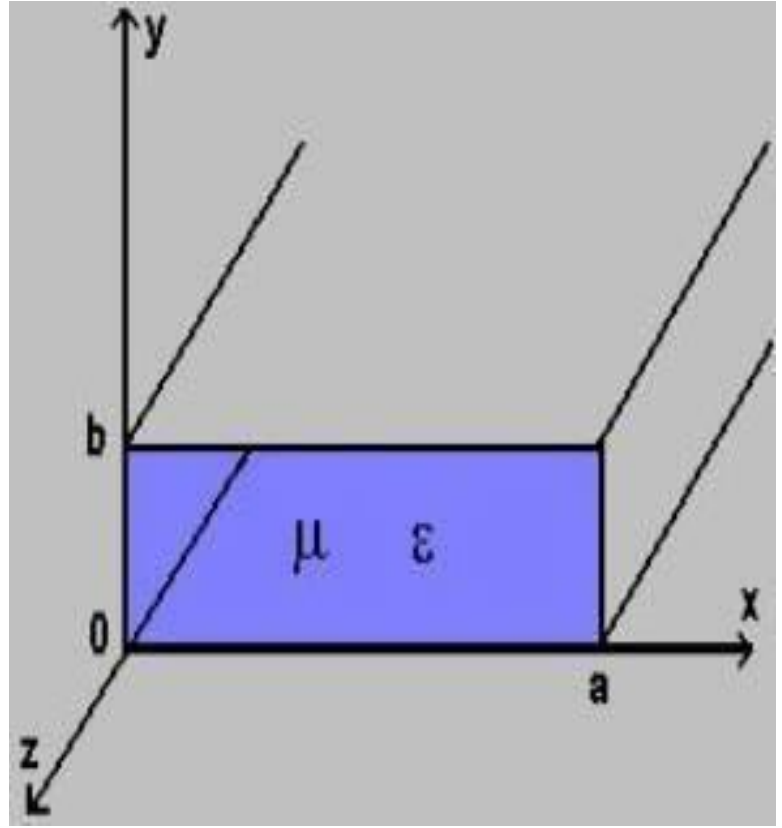

Fig 1: Rectangular Waveguide coordinate system

The cutoff frequency for different modes in a rectangular waveguide is given as:

$$
\mathrm{f}_{c}=\frac{1}{2 \sqrt{\mu \varepsilon}} \sqrt{\frac{m^{2}}{a^{2}}+\frac{n^{2}}{b^{2}}}
$$

$\mathrm{m}, \mathrm{n}=0,1,2 \ldots$

Where $\mathrm{a}$ and $\mathrm{b}$ are the dimensions of the waveguide, which has already been specified, and since the medium is air the permittivity is 1 .

The guided wavelength, which is defined as the distance between the two equal phase planes along the waveguide, is given as:

$$
\lambda g=\frac{\lambda}{\sqrt{1-(f c / f)^{2}}} \text { for } f>f_{c}
$$

The propagation constant gamma is given as:

Where,

$\alpha=$ attenuation constant $\beta=$ phase constant

\section{THEORETICAL EXPECTED}

\section{RESULTS}

The cutoff frequencies for various modes are calculated using equation 3 for different modes and the expected results for the different modes are shown below:

Table1. TMM,N Cutoff Frequency

\begin{tabular}{|l|l|l|}
\hline$M$ & N & $\mathrm{Fc}_{\mathrm{m}, \mathrm{n}}(\mathrm{GHz})$ \\
\hline 1 & 1 & 16.156 \\
\hline 1 & 2 & 30.248 \\
\hline 2 & 1 & 19.753 \\
\hline
\end{tabular}

Table2. TEM,N Cutoff Frequency

\begin{tabular}{|l|l|l|}
\hline$M$ & N & $\mathrm{Fc}_{\mathrm{m}, \mathrm{n}}(\mathrm{GHz})$ \\
\hline 1 & 0 & 6.562 \\
\hline 2 & 0 & 13.123 \\
\hline 0 & 1 & 14.764 \\
\hline 1 & 1 & 16.156 \\
\hline
\end{tabular}

The dominant mode in TE and TM modes are clearly $\mathrm{TE}_{10}$ and $\mathrm{TM}_{11}$.

\section{SIMULATIONS AND RESULTS}

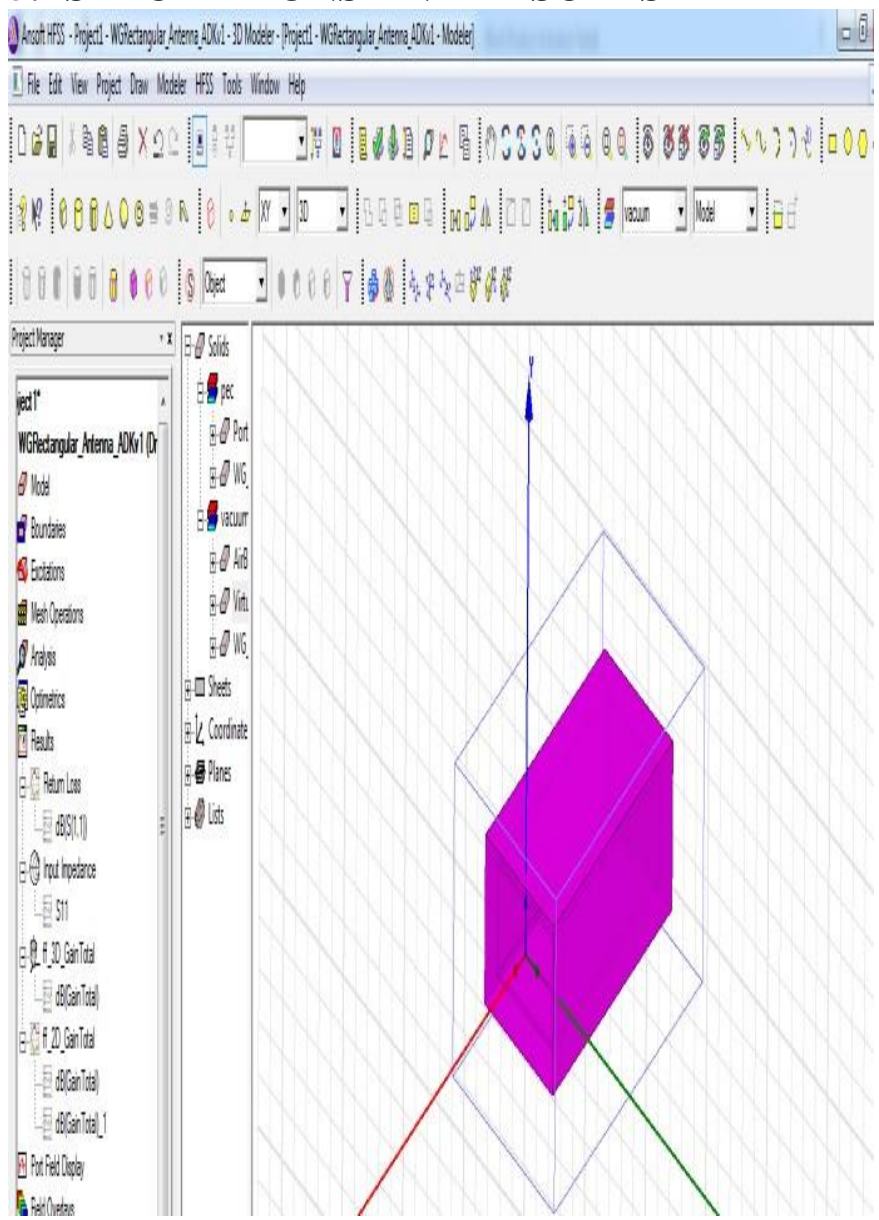

Fig 2: Rectangular waveguide simulation by HFSS

The propagation constant vs frequency plot is plotted for the four modes and the result is expected with the theoretical result as shown in the figure 3:

Here we can see that the 4 modes $\mathrm{TE}_{10}, \mathrm{TE}_{20}, \mathrm{TE}_{01}$ and $\mathrm{TM}_{11 \text { are }}$ simulated against the propagation constant and the resultis in accordance with the theoretical value. The cutoff value for different modes is in accordance with the theoretical values. A $3 \mathrm{D}$ plot is also plotted for the same as shown in figure 4 : 


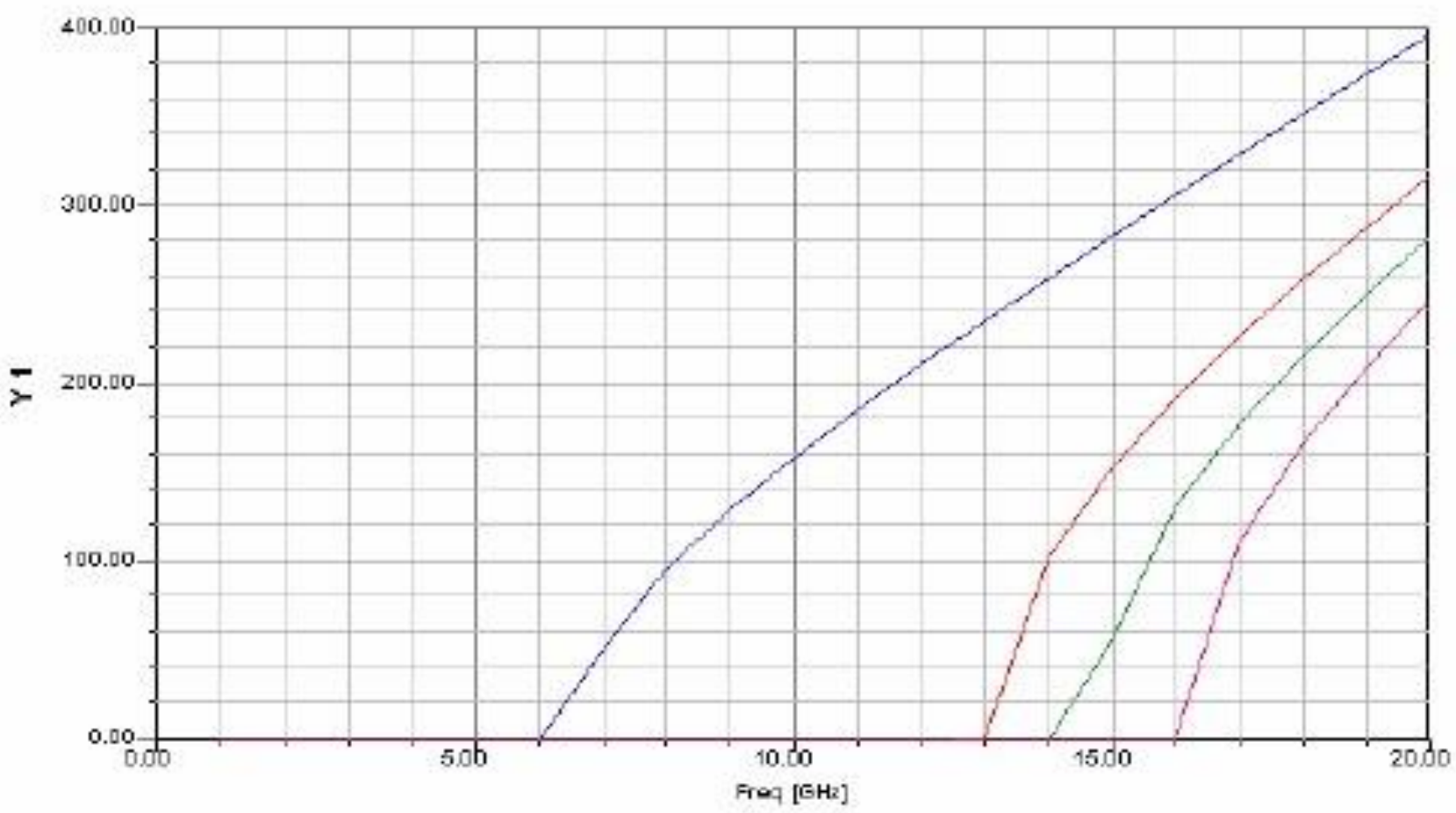

Fig 3: Plot of Frequency vs Propagation Constant

The Electric and magnetic field intensity patterns have also been plotted. The electric field intensity decreases from top to bottom. Figure 5 and 6 show the electric and magnetic field intensity.

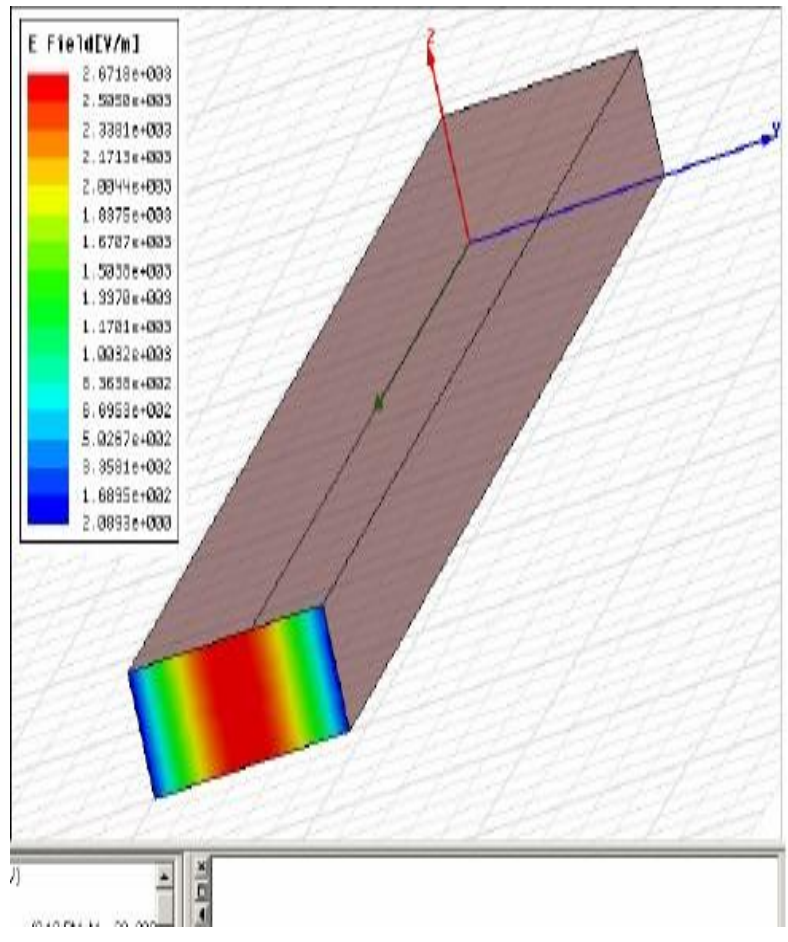

Fig4: 3D Rectangular plot for cut off frequency for different modes

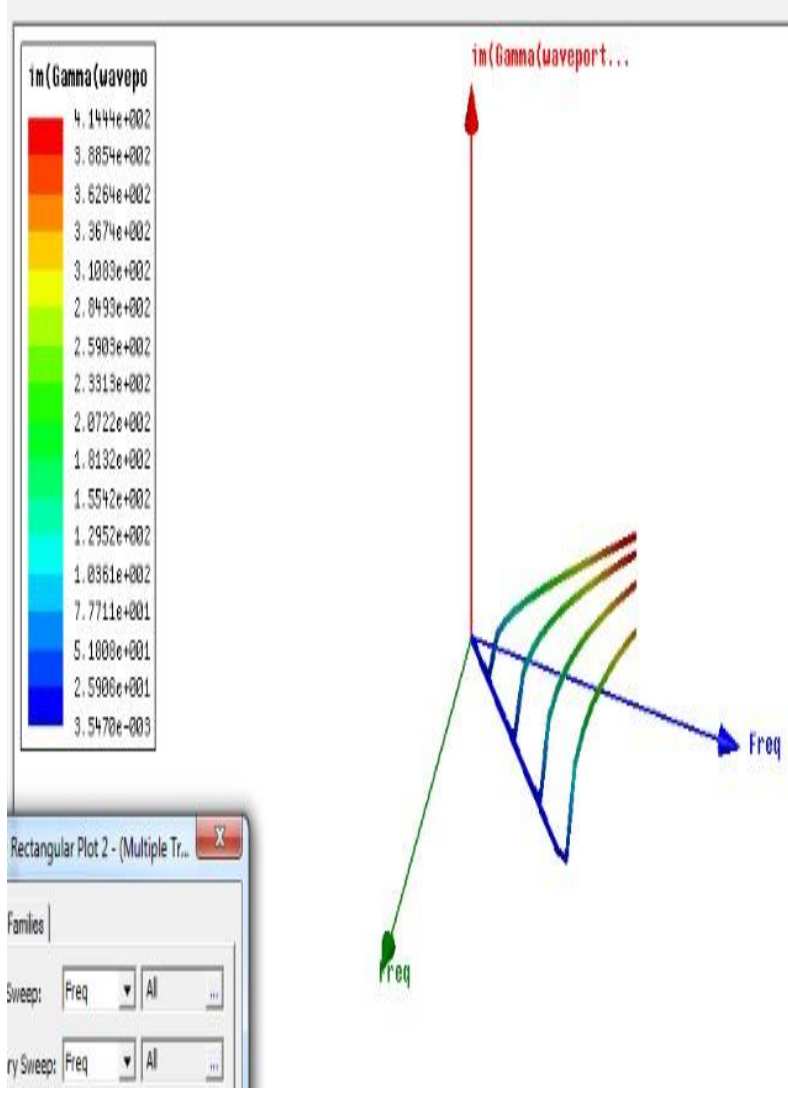

Fig5: Electric Field Intensity in a Rectangular Waveguide 


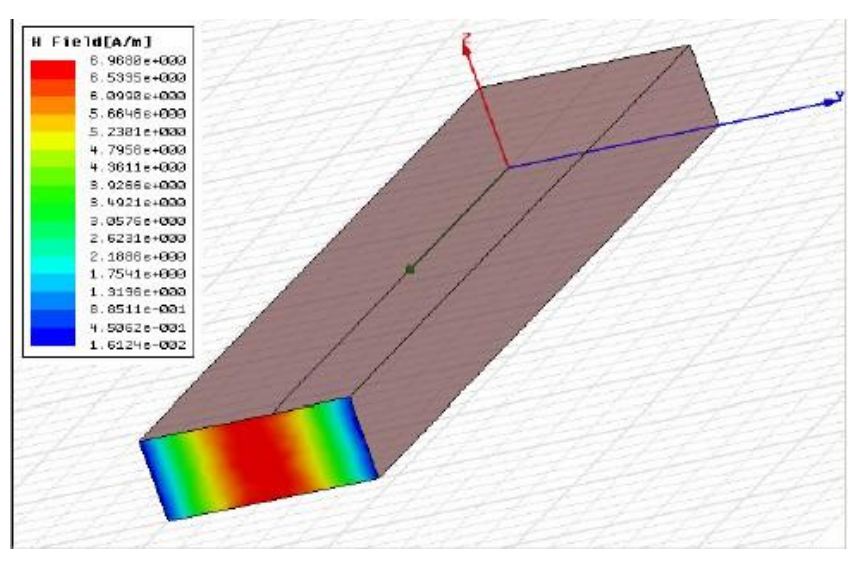

Fig 6: Magnetic field intensity in a rectangular waveguide

The colors indicate the intensity of the field decreasing from top to bottom.

\section{CONCLUSION}

A rectangular waveguide is simulated using HFSS and an excellent match between the simulated and theoretical values has been found. The cutoff frequency calculated theoretically matches with the cutoff frequency found out by the simulation. The properties of waveguide have been used to determine the characteristics of the waveguide. The frequency used for sweep is used more than the highest frequency of the cutoff of the last mode. The Electric as well as the Magnetic Field Patterns have been simulated and the results match with the theoretical analysis.

These simulations have been carried out for experimental analysis and can be more helpful before the designing of real world components.

\section{REFERENCES}

[1] S.Paramesha and A, Chakraborty "Waveguide as a nearfield measuring probe of the two element array radiator" Progress in Electromagnetics Research PIER B, vol. 7,245-255, 2008.

[2] Jiao, C. Q," Selective suppression of Electromagnetic Modes in a Rectangular Waveguide by Distributed Wall Losses", Progress in Electromagnetics Research PIER letters, vol. 22, pp. 119-128, 2011.

[3] Lee, C. S, Lee, S. W. and Chuang, S. L, "plot of modal field distribution in rectangular and circular waveguides", IEEE transactions on microwave theory and techniques, vol. 33, pp.271-274, 1985.

[4] Simon, w, wert hen, Mandy wolf F, I, "A novel coplanar transmission line to rectangular waveguide transition,' IEEE MTT-society international symposium digest. Pp .257-260, 1998.

[5] Samuel Y Liao, 2008, "Microwave Devices and Circuits" 3rd Edition Pearson Prentice Hall.

[6] Harrington, R. F, "Time harmonic Electromagnetic Fields," McGraw- Hills, New York, 1961.

[7] C, A Balanis "Advanced Engineering Electromagnetic," 1989, John Wiley and Sons Inc.

[8] R.E Collin, "Foundation for microwave engineering",2nd edition, 1992.McGraw-Hills New York.

[9] Baudrand, H, Tao, J,W, Atechian, J ."Study of radiating properties of open ended rectangular wave guides", Antennas and Propagation, IEEE Transactions on pages; 1071-1077 Vol;36, Issue;8,Aug. 1988. 